\title{
Surface deformation analysis of collapsed lungs using model-based shape matching
}

\author{
Megumi Nakao ${ }^{1}\left[\right.$ ] Junko Tokuno ${ }^{2} \cdot$ Toyofumi Chen-Yoshikawa $^{2} \cdot$ Hiroshi Date $^{2} \cdot$ Tetsuya Matsuda $^{1}$
}

Received: 16 January 2019 / Accepted: 5 June 2019 / Published online: 27 June 2019

(c) The Author(s) 2019

\begin{abstract}
Purpose To facilitate intraoperative localization of lung nodules, this study used model-based shape matching techniques to analyze the inter-subject three-dimensional surface deformation induced by pneumothorax. Methods: Contrast- enhanced computed tomography (CT) images of the left lungs of 11 live beagle dogs were acquired at two bronchial pressures (14 and $2 \mathrm{~cm} \mathrm{H}_{2} \mathrm{O}$ ). To address shape matching problems for largely deformed lung images with pixel intensity shift, a complete Laplacian-based shape matching solution that optimizes the differential displacement field was introduced.

Results Experiments were performed to confirm the methods' registration accuracy using CT images of lungs. Shape similarity and target displacement errors in the registered models were improved compared with those from existing shape matching methods. Spatial displacement of the whole lung's surface was visualized with an average error of within $5 \mathrm{~mm}$.

Conclusion The proposed methods address problems with the matching of surfaces with large curvatures and deformations and achieved smaller registration errors than existing shape matching methods, even at the tip and ridge regions. The findings and inter-subject statistical representation are directly available for further research on pneumothorax deformation modeling.
\end{abstract}

Keywords Pneumothorax deformation analysis · Model-based shape matching $\cdot$ Lung $\cdot$ Thoracoscopic surgery

\section{Introduction}

Recent advances in medical imaging technology have enabled visualization of early stage cancer, metastatic lung tumors, and benign nodules. Video-assisted thoracoscopic surgery $[1,2]$ is a widely performed minimally invasive surgical procedure. Although lung nodules are examined on preoperative computed tomography (CT) images during preoperative planning, the position of a nodule may change because of the state of pneumothorax during surgery, which makes optimization of resection procedures difficult. Although various attempts have been made to use physical or chemical markers $[3,4]$ to identify the varying positions of multiple nodules,

Electronic supplementary material The online version of this article (https://doi.org/10.1007/s11548-019-02013-0) contains

supplementary material, which is available to authorized users.

Megumi Nakao

megumi@i.kyoto-u.ac.jp

1 Graduate School of Informatics, Kyoto University, Yoshida-Honmachi, Sakyo-ku, Kyoto, Japan

2 Department of Thoracic Surgery, Kyoto University Hospital, 54 Kawaharacho, Shogoin, Sakyo-ku, Kyoto, Japan the associated clinical burdens on both surgeons and patients are increased because of the additional CT imaging and preoperative marking procedures required. If the intraoperative positions of lung nodules could be accurately estimated, precise nodule resection and preservation of pulmonary function could be facilitated by the strict management of resection margins.

Deformable image registration techniques [5-7] for the analysis of organ and soft tissue deformations have been explored previously. Intraoperative deformation due to changes in internal pressure, patient posture, and tool manipulation is a well-known practical registration problem, which must be addressed in the development of intraoperative guidance systems [8-16]. Specifically, in the field of imagebased lung modeling, respiratory motion has been the main focus of investigation [17-21]. However, there have been few studies on modeling of the pneumothorax deformation that occurs between the preoperative and intraoperative lung states. Shape matching of the pneumothorax deformation of the lung should address the technical issues of the large deformations and CT intensity shifts that occur. Lungs are very soft organs, and their deformation can induce considerable volume change. The mechanism of pneumothorax deforma- 
tion is complex and not mathematically understood, except through simulation studies of animal lungs [17,22]. CT intensity shifts occur in the atelectasis state, where the air content of the lungs is reduced, resulting in changes to CT values and lowering of image contrast, and reduced performance for image-based registration.

To localize lung nodules during surgery, Nakamoto et al. [23] proposed intraoperative registration methods that matched the surface of a preoperative CT model with several surface points optically measured on the deflated lungs. Intraoperative segmentation of thoracoscopic camera images was investigated in [24], and Alvarez et al. recently reported deformable registration results for lung deformations resulting from postural differences on cone beam CT (CBCT) [25]. Uneri et al. also proposed a registration framework to analyze the displacement of internal lung structures on CBCT data from animal lungs [26]. To address CT intensity shifts, an integrated framework of model-based and image-based registration was used; however, registration of the bronchial branch points was the main focus, and registration accuracy at the lung surface was not evaluated.

The goals and clinical needs of intraoperative guidance using video-assisted thoracic surgery (VATS) are visualization of the subsurface positions of nodules, bronchial structures, and vascular structures in the collapsed lungs. However, it is difficult to image internal structures with VATS, because CBCT imaging is not available in most clinical facilities, and it may increase the burden on patients and surgeons. Recent studies [12,27] proposed using the 2D appearance or silhouettes of organs as visual cues to register preoperative $\mathrm{CT}$ models with intraoperative camera images. We also consider that the intraoperative appearance of collapsed lung surfaces could be an essential visual cue $[28,29]$. Clinical applications of surface deformation models are widespread and include initial model alignment and 2D-3D surface matching, e.g., matching of 2D intraoperative camera images (2D surface or silhouettes) with volumetric deformation models (3D surface with subsurface structures) of the collapsed lungs. Integrating surface and subsurface deformation models into a thoracoscopic camera image recognition system could lead to novel vision-based guidance for VATS, which could provide subsurface nodule visualization without additional CBCT measurement. However, to the best of our knowledge, no study has shown such registration results, or visualized inter-subject variation in spatial deformation of whole lung structures. Specifically, registration error tends to increase in areas with substantial curvature, such as the tips of the lobes or the boundary region between the upper and lower lobes. Therefore, data acquisition and detailed surface analysis is worth investigating in the search for a statistical formulation of lung deformation.

In this study, we used model-based shape matching to analyze three-dimensional surface displacement in collapsed lung. First, CT datasets were acquired at two bronchial pressures (assigned as the inflated and deflated states) in 11 live beagle dogs. Although spatial distribution of the displacement of internal structures can be obtained by the matching of anatomical feature points such as bronchial branches $[23,26]$, landmark-based matching cannot be applied to curved surfaces, because of their lack of anatomical features. As the acquired CT data may contain large deformations with CT intensity shifts, global image registration techniques [30] tend to lead to large registration errors, particularly at tip regions with high curvature.

The practical applications of model-based registration are increasing, as registered mesh models are directly available for statistical modeling and variational analysis [31-33]. In addition, a recent study [32] reported that registration accuracy for anatomical structures with large shape variations was better than that obtained using large deformation diffeomorphic metric mapping [7]. Here, we employ a model-based shape matching approach utilizing improved Laplacianbased shape matching (LSM) techniques for pneumothorax deformation. We performed experiments to confirm the performance of the proposed shape matching method using CT data and to summarize the spatial distribution of the three-dimensional displacement of the lung surface during pneumothorax deformation.

The contributions of this paper are: (1) a complete model-based surface registration solution that optimizes a differential displacement field to map large pneumothorax deformations and (2) analysis of the spatial displacement of whole inflated/deflated lungs, including statistical deformation model representations. This paper does not focus on discussing the technical aspects of the registration methods, but instead concentrates on reporting the findings of pneumothorax deformation analysis with application of improved model-based shape matching.

\section{Methods}

\section{Measurements and surface reconstruction}

To analyze the deformation of collapsed lung, contrastenhanced CT images of the left lungs of 11 live beagle dogs were acquired at two bronchial pressures ( 14 and $2 \mathrm{~cm} \mathrm{H}_{2} \mathrm{O}$ ) at the Institute of Laboratory Animals, Kyoto University. This study was performed in accordance with the regulations of the Animal Research Ethics Committee of Kyoto University. All CT images were acquired on a 16-row multidetector CT scanner (Alexion 16, Toshiba Medical Systems, Tochigi, Japan). During the procedure, the dogs were maintained under anesthesia with ketamine, xylazine, and rocuronium and underwent tracheal intubation and mechanical ventilation by a ventilator (Savina 300, Drager AG \& Co. KGaA, 


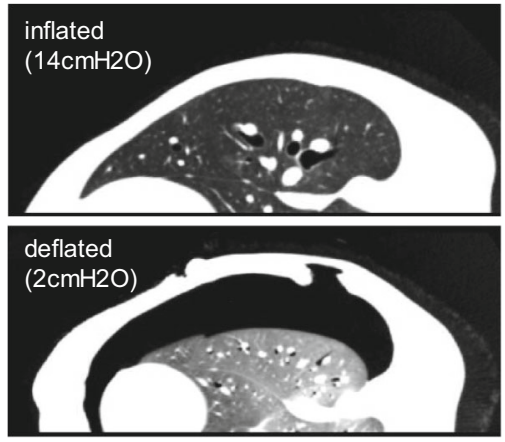

Fig. 1 CT images of inflated/deflated states with intensity shift measured from the left lung of a live beagle dog

Lübeck, Germany). A single trocar hole was first made on the chest wall, to let air flow into the pleural cavity. Using the ventilator, the bronchial pressure was set to $14 \mathrm{cmH}_{2} \mathrm{O}$ to obtain images of the fully expanded lungs (inflated state), and to $2 \mathrm{cmH}_{2} \mathrm{O}$ for imaging of the collapsed lung state (deflated state). All dogs were placed in a right lateral (decubitus) position on the bed of the CT scanner, and the two CT image sets of the inflated and deflated states were acquired in that order for each dog. For the contrast-enhanced CT, $10 \mathrm{~mL}$ of iopamidol contrast agent was injected through a lower extremity peripheral vein. Scanning was performed $5 \mathrm{~s}$ after the injection of iopamidol.

Figure 1 shows CT slices from the inflated and deflated states after registering the two volumes using the spine as a fixed reference. The CT intensity values change because of differences in the air content of the lung, with the CT intensity values of the parenchyma increasing (the parenchyma region becomes brighter) in the deflated state. This intensity shift can be clearly observed in the lower image of Fig. 1 when it is compared with the upper image. For details, refer to [26]. The registration accuracy of image-based registration can be affected by these imaging characteristics of pneumothorax deformation, as most of the methods assume consistent pixel features. The proposed model-based registration approach achieves stable registration for pneumothorax deformation analysis on the basis of surface geometry.

Anatomical segmentation of the upper and lower lobes was automatically performed using the Synapse VINCENT image analysis system (Fujifilm Co., Ltd.), and the surfaces of the lobes were generated as triangulated mesh representations using Poisson surface reconstruction [34]. The meshes of the two lobes were created independently, and each triangulated mesh was stored in the standard STL or PLY file format.

\section{Shape matching between inflated and deflated lungs}

To calculate the lung surface displacement, shape matching was performed on the triangulated meshes reconstructed
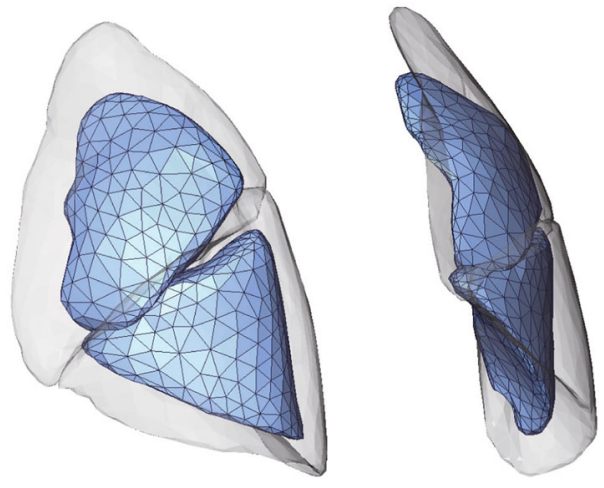

Fig. 2 Example of reconstructed lung surfaces in inflated and deflated states. The translucent image depicts the inflated state, and the opaque image with the mesh topology is the deflated state

from the CT images. Figure 2 shows an example of reconstructed lung surfaces in the inflated and deflated states, and Fig. 3 outlines the inter-subject shape matching framework developed using the statistical motion modeling expression [33]. A template surface $T$ was used as the source, and the individual surfaces $S_{I}^{(k)}(k=1,2, \ldots, n)$ in the inflated and $S_{D}^{(k)}(k=1,2, \ldots, n)$ deflated states were used as the targets. In this study, $n$ equals 11 , because we prepared an image dataset with 11 subjects in the inflated and deflated states. Here, we assume that the pair of surfaces $\left(S_{I}^{(k)}\right.$ and $S_{D}^{(k)}$ ) differs in the number of vertices and the structure of the mesh (i.e., mesh topology), as they were independently generated from different CT images. As shown in Fig. 3a, the corresponding models $M_{I}^{(k)}, M_{D}^{(k)}$ (with the same vertex and the same mesh topology) that precisely approximated the surfaces $S_{I}^{(k)}$ and $S_{D}^{(k)}$, respectively, are computed by shape matching. Because the two registered models achieve point-to-point correspondence, spatial deformation $D^{(k)}=$ $M_{D}^{(k)}-M_{I}^{(k)}$ can be represented by calculating the displacement vector of the corresponding vertex, as shown in Fig. $3 \mathrm{~b}$. To capture rotational components or the sliding motion of the upper and lower lobes, this registration process is applied to each lobe independently. Unlike per-subject registration, our approach enables the construction of a statistical deformation model, making deformation analysis among subjects possible.

For the template generation, one case was randomly selected and set as the initial surface for $T$, and the triangular surface was resampled to 400 vertices and 796 triangles for each lobe. Next, the corresponding models $M_{I}^{(k)}$ were obtained by registering $T$ to the individual surfaces $S_{I}^{(k)}$ in the inflated state. As the mesh models $M_{I}^{(k)}$ have point-topoint correspondence, the average shape $\bar{M}$ can be obtained by calculating the average of each coordinate. We used $\bar{M}$ as the final template. By keeping the template close to the data to be matched in advance, we aimed to reduce the influence of 
Fig. 3 Outline of the inter-subject shape matching framework. a Corresponding models $\left(M_{I}^{(k)}, M_{D}^{(k)}\right)$ with the same vertex and the same mesh topology are computed by registering the template $T$ to the individual surfaces $\left(S_{I}^{(k)}, S_{D}^{(k)}\right)$. b The spatial deformation $D^{(k)}$ between the inflated state and deflated state is obtained from the registration between the two models

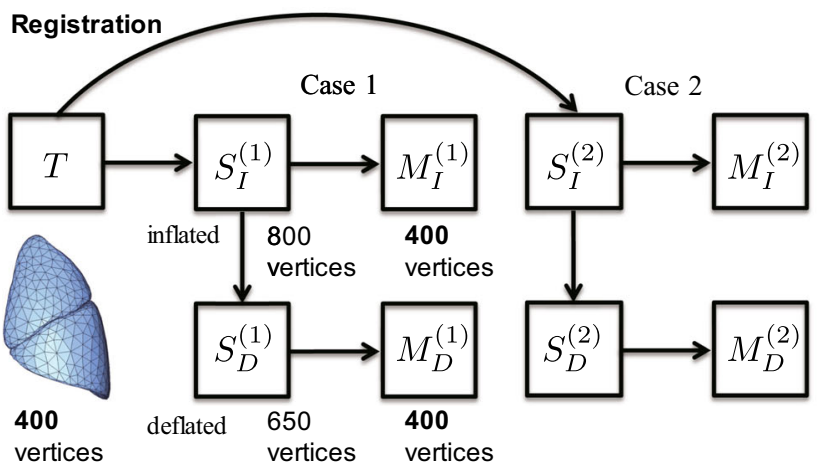

(a)

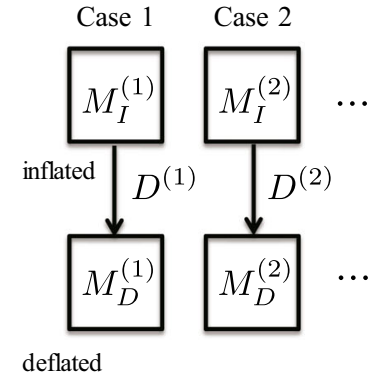

(b) the template shape's data selection method on the matching, while preventing increased matching error.

Accurate shape matching is required to compute a stable three-dimensional displacement field from the substantially deformed surfaces. As pneumothorax deformation includes considerable volume changes and rotations, the registration error of image-based global registration methods increases in areas with large curvature, such as the tips of the lobes or boundary region between the upper and lower lobes. To achieve both globally stable and locally strict registration, Laplacian-based shape registration is used after executing affine transformation. A discrete Laplacian was first introduced for interactive editing of a geometric model [35] and was recently applied to non-rigid shape modeling. In [32], Laplacian-based registration showed better registration performance than large deformation diffeomorphic metric mapping [7] for curved surfaces with shape variations. Lung surfaces also move considerably during pneumothorax deformation, and the registration accuracy is not understood. Therefore, in this research, we extended the Laplacian-based registration technique to shape matching of inflated/deflated lungs with large-scale deformations and investigated its registration performance.

\section{Laplacian-based surface registration using a differential displacement field}

The overall process of the shape matching framework developed is described as follows.

STEP 1 A discrete Laplacian $L\left(\boldsymbol{v}_{i}\right)$ and a normal vector $\boldsymbol{n}_{i}$ are calculated for all vertices $\boldsymbol{v}_{i}$ of the template $T$ and the target surface $S$.

STEP 2 Localized shape similarity $Q_{i}$ between the template and the target surface is calculated for all vertices of the template mesh.

STEP 3 The positional constraint $\boldsymbol{p}_{i}$ for shape update is determined based on Eq. (4).
STEP 4 The new positions for the set of vertices $\boldsymbol{v}_{i}^{\prime}$ are calculated based on Eq. (2). The shape is updated as $\boldsymbol{v}_{i} \leftarrow \boldsymbol{v}_{i}^{\prime}$. Then, back to STEP 1 .

The discrete Laplacian obtained in STEP 1 is a shape descriptor defined as Eq. (1) that approximates the mean curvature normal of the triangular mesh.

$L\left(\boldsymbol{v}_{i}\right)=\sum_{j \in N\left(\boldsymbol{v}_{i}\right)} \omega_{i j}\left(\boldsymbol{v}_{i}-\boldsymbol{v}_{j}\right)$

Here, $\omega_{i j}$ is a weight, and $N\left(\boldsymbol{v}_{i}\right)$ is the number of adjacent vertices of one ring connected by the vertex $\boldsymbol{v}_{i}$ and the edge.

$\hat{V}=\underset{V}{\arg \min } \sum_{i=1}^{n}\left\|L\left(\boldsymbol{v}_{i}^{\prime}\right)-L\left(\boldsymbol{v}_{i}\right)\right\|^{2}+\delta \sum_{i=1}^{n}\left\|\boldsymbol{p}_{i}-\boldsymbol{v}_{i}\right\|^{2}$

where $V$ is the set of vertices $\boldsymbol{v}_{i}$ at their initial positions, and $V^{\prime}$ is the set of vertices $\boldsymbol{v}_{i}^{\prime}$ to be solved. $\boldsymbol{p}_{i}$ is a positional constraint set to $\boldsymbol{v}_{i}$, and $\delta$ is a weight parameter configured according to the problem. $L(\cdot)$ is the Laplace-Beltrami operator, and $L\left(\boldsymbol{v}_{i}\right)$ is the discrete Laplacian at the vertex $\boldsymbol{v}_{i}$. The first term is a penalty to shape changes to the mesh, and the second term increases if the constrained vertex is distant from the target position $\boldsymbol{p}_{i}$. By computing $\boldsymbol{v}_{i}^{\prime}$, which minimizes the objective function, the template model is updated while preserving the shape as much as possible. Because Eq. (2) is a quadratic minimization problem at vertex positions $\boldsymbol{v}_{i}$, it is possible to calculate it with a low computation cost.

Here, we describe the method of determining the positional constraints $\boldsymbol{p}_{i}$ in STEP 2 and STEP 3. It is difficult to find appropriate positional constraints in the initial state when the deformation between surfaces is large and anatomical landmarks and point-to-point correspondences are not given. Therefore, we explored better constraint options by performing a progressive search and additionally introduced a new definition of positional constraints that enabled us to 
obtain smooth displacement while performing precise surface matching. To obtain the three-dimensional position on the target model corresponding with the vertex of the template model, the shape similarity value $Q$ was calculated for all combinations of the vertex $v_{s}$ of the template model and the vertex $v_{t}$ of the target model. $Q$ is defined by (3)

$$
Q\left(\boldsymbol{v}_{s}, \boldsymbol{v}_{t}\right)=\left|\boldsymbol{v}_{s}-\boldsymbol{v}_{t}\right|+\gamma\left(1-\boldsymbol{n}_{s} \cdot \boldsymbol{n}_{t}\right)
$$

where $\gamma$ is a weight, and $\boldsymbol{n}_{s}, \boldsymbol{n}_{t}$ are vertex normals in $\boldsymbol{v}_{s}, \boldsymbol{v}_{t}$, respectively. $Q$ locally evaluates shape similarity per vertex, and if $Q$ is small enough, the pair of tangent planes expressed by $\boldsymbol{v}_{s}$ and $\boldsymbol{v}_{t}$ is corresponding surfaces.

The positional constraints $\boldsymbol{p}_{i}$ are configured on the basis of the local similarity $Q$ for all vertices of the template model. For a group of vertices whose $Q$ is small enough, or close to zero, the positions of vertices $\boldsymbol{v}_{s}$ are maintained, that is, $\boldsymbol{p}_{i}=\boldsymbol{v}_{s}$ is given, because they adequately represent the local surface of the target shape. Alternatively, for a group of vertices with a large $Q$ value, a positional constraint is set to correct the local shape of the template. In this case, if the corresponding point is determined independently for each vertex of the template model, the progressive shape update may yield a non-smooth displacement field. Figure 4a briefly illustrates the issue of local matching error caused by independently configured positional constraints. To address this problem, we focused on the differential displacement field $\nabla \boldsymbol{u}$ and achieved smooth deformation while performing precise surface matching. Figure $4 \mathrm{~b}$ provides an example of the modified positional constraints obtained after shape matching.

The differential displacement field is computed by the set of partial differentials of displacements $\boldsymbol{u}_{s}$ on the vertex $\boldsymbol{v}_{s}$. To smooth the displacement field, the changes in the gradients of the displacement field $\boldsymbol{u}_{s}$ should be kept small, as shown in Fig. 4c. This means that smooth deformation matching between two shapes can be performed by minimizing the Laplacian of the displacements $L\left(\boldsymbol{u}_{s}\right)$. This concept is based on Laplacian-based mesh optimization [36], which achieves feature-preserving smoothing of triangular meshes. The different aspects are that this strategy (1) applies its basic technique to the displacement field, not to the surface, and (2) undergoes progressive updates throughout the shape matching process, which aims to solve the trade-off problems of the nearest neighbor search between point-to-point correspondences and maintenance of a smooth displacement field.

On the basis of this scheme, when the pair of corresponding vertices $\left(\boldsymbol{v}_{s}, \boldsymbol{v}_{t}\right)$ is determined by the local similarity index $Q$, the positional constraint $\boldsymbol{p}$ is determined as an internal division point of the smoothed position $\boldsymbol{v}_{s}^{\prime}$ and its projected position $\boldsymbol{v}_{t}^{\prime}$ on the target tangent plane. Figure $4 \mathrm{~d}$ illustrates the setup of positional constrains $\boldsymbol{p}_{i}$ from the pair of vertices $\left(\boldsymbol{v}_{s}, \boldsymbol{v}_{t}\right)$. This step-by-step update avoids local mismatch at the early stage assuming a considerable distance between the two surfaces. Consequently, the positional constraints $\boldsymbol{p}_{i}$ are defined by Eq. (4).

$\boldsymbol{p}_{i}= \begin{cases}\boldsymbol{v}_{s}^{\prime}+\frac{l_{s}}{m}\left(\boldsymbol{v}_{t}^{\prime}-\boldsymbol{v}_{s}^{\prime}\right) & \left(Q>Q_{\text {high }}\right) \\ \boldsymbol{v}_{s}^{\prime} & \left(Q<Q_{\text {low }}\right)\end{cases}$

$\boldsymbol{v}_{s}^{\prime}=\boldsymbol{v}_{s}-\lambda L\left(\boldsymbol{u}_{i}\right)$

$L\left(\boldsymbol{u}_{i}\right)=\sum_{j \in N\left(\boldsymbol{v}_{i}\right)} \omega_{i j}\left(\boldsymbol{u}_{i}-\boldsymbol{u}_{j}\right)$

Here, $l_{s}$ is the average length of all edges connected to the vertex $\boldsymbol{v}_{i}$, and $m$ is a step constant for the progressive search. The two threshold values $Q_{\text {high }}$ and $Q_{\text {low }}$ affect the stability of the shape update and the convergence speed. For example, if a high value is used for $Q_{\text {high }}$, a smaller number of vertices are constrained at the target surface, which results in slower convergence. When a low value is used for $Q_{\text {low }}$, the template shape becomes easily deformable, possibly destabilizing the shape updating, as a smaller number of vertices are fixed. In our case, after investigating various parameter sets, the average value of the top $10 \%$ was used as $Q_{\text {high }}$, and the average value of the bottom $2 \%$ was used as $Q_{\text {low }}$.

In STEP 4, the template surface is updated by applying the positional constraint $\boldsymbol{p}$ to the quadratic minimization equation (2). The shape optimization defined by STEP 1 to STEP 4 is iteratively processed. When the maximum value of the inter-surface distance between the template and the target model is not improved when the surface update is repeated ten times, or when the number of updates reaches 3000 times, the iterative process is terminated. In this framework, even when the number of vertices differs between the template and target models, or even when the vertex of the target model does not exist near to the corresponding local region, the optimized matching of corresponding local surfaces can be computed.

\section{Experiments and results}

In the experiments, the performance of the proposed Laplacian-based shape matching framework was first evaluated. The efficacy of the differential displacement field was confirmed by comparing it with existing shape registration methods. Then, the pneumothorax deformation of lungs was investigated in terms of the linearity and morphological variation of deformation using the registered models. The proposed shape matching framework was implemented using Visual $\mathrm{C} / \mathrm{C}++$ and OpenGL. A computer with a graphics processing unit (CPU: Intel Core i7 $3.7 \mathrm{GHz}$, Memory: $64 \mathrm{~GB}$, GPU: NVIDIA GeForce GTX 1080) was used throughout the experiments. For the weights in the developed framework, we 


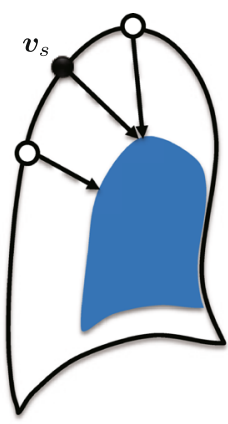

(a)

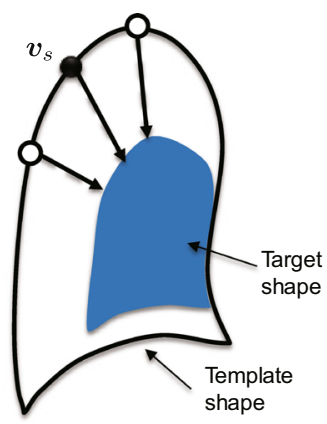

(b)

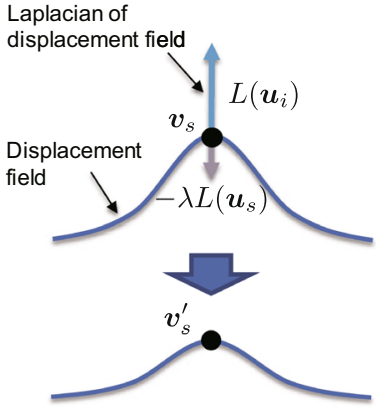

(c)

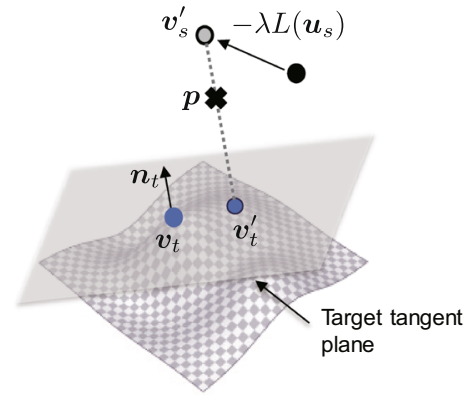

(d)
Fig. 4 Positional constraints for Laplacian-based surface registration to ensure a smooth differential displacement field. When the corresponding pair of vertices $\left(\boldsymbol{v}_{s}, \boldsymbol{v}_{t}\right)$ is given, the positional constraint $\boldsymbol{p}$ is determined as an internal division point of the smoothed position $\boldsymbol{v}_{s}^{\prime}$ and its projected position $\boldsymbol{v}_{t}^{\prime}$ on the target tangent plane used 10.0 for $\delta$, and 1.0 for $\omega, \delta$ and $\gamma$ after examination of several parameters sets.

\section{Performance evaluation}

Shape matching was performed on 11 in vivo lung models (Cases 1-11) to confirm registration accuracy. The procedure for creating the template $T$ and lung mesh models from the in vivo CT images is described in "Measurements and surface reconstruction section." Table 1 summarizes the volumes of the upper/lower lobes at bronchial pressures of 14 and $2 \mathrm{cmH}_{2} \mathrm{O}$ for each case, with $V$ being the volume. For the superscript/subscript characters, $u$ : upper lobe, $l$ : lower lobe, $i$ : inflated state, and $d$ : deflated state. $r$ is the ratio of volume change: $V^{d} / V^{i}$. We note that the volume of Case 11 at a bronchial pressure of $2 \mathrm{cmH}_{2} \mathrm{O}$ was unexpectedly increased, which is probably a result of the bronchial pressure not being successfully controlled. However, as shape matching can be applied to such an inflated shape, the performance of the shape matching was evaluated for all the datasets.

\section{Quantitative comparisons of shape matching}

In this study, the mean distance [32], Hausdorff distance [37], and Laplacian of the displacement were used as the shape similarity criteria. The Hausdorff distance measures the longest distance among minimum point distances between two surfaces, whereas the mean distance is the average of the minimum point distances. Unlike segmentation or recognition problems, shape matching requires point-topoint correspondence between two shapes. For example, as the Dice coefficient only measures volume overlap, it is not sufficient to evaluate per-vertex correspondence, nor to measure the quality of local matching. The Laplacian of the displacement is the magnitude of the second derivatives of the displacement field and evaluates the smoothness of the deformation. The registration accuracy was compared among four shape matching approaches: (1) LSMD: the proposed Laplacian-based shape matching with optimization of the differential field defined in this paper, (2) LSM: Laplacianbased shape matching with a similar progressive deformation approach used in [32] and [38], (3) PWA: piecewise affine transformation [39], and (4) AF: affine transformation. For all algorithms, affine transformation was performed in advance, to globally match the posture and volume of the entire shape.

Figure 5 shows box plots of the mean distance, Hausdorff distance, and mean and maximum Laplacian of the displacement computed from the 11 subjects' registration results. Figure 5a, b shows the results for upper lobes and lower lobes, respectively. The box plots include the minimum, first quartile $(Q 1)$, median $(Q 2)$, third quartile $(Q 3)$, and maximum. The minimum and maximum scores are represented after outliers were rejected. Values larger than $(Q 3-Q 1) \times 1.5+Q 3$ or smaller than $Q 1-(Q 3-Q 1) \times 1.5$ were regarded as outliers. The average, minimum, and maximum values of all datasets are summarized for LSMD, LSM, PWA, and AF in Table 2.

LSMD and LSM achieved a significantly smaller mean distance than the other two methods, and a Hausdorff distance with an error within $1 \mathrm{~mm}$; they therefore outperformed the other two methods in terms of matching volumetric regions. Regarding the Laplacian of the displacement field in the LSMD and LSM methods, in the right of Fig. 5, the LSDM had smaller values than LSM, which means that a smooth deformation with reduced unstable surface matching can be performed using LSMD.

\section{Target displacement error}

In addition to the above geometrical indices, the accuracy of displacement vectors obtained after shape matching was 
Table 1 Volumes of upper/lower lobes on CT images of the left lungs of live beagle dogs. $V$ is the volume

\begin{tabular}{lllllll}
\hline Case & $V_{u}^{i}[c c]$ & $V_{u}^{d}[c c]$ & $r_{u}[\%]$ & $V_{l}^{i}[c c]$ & $V_{l}^{d}[c c]$ & $r_{l}[\%]$ \\
\hline 1 & 173.5 & 82.7 & 47.7 & 242.9 & 96.9 & 39.9 \\
2 & 167.1 & 69.9 & 41.8 & 508.3 & 116.4 & 22.9 \\
3 & 231.7 & 111.4 & 48.1 & 357.6 & 145.3 & 40.6 \\
4 & 184.7 & 71.4 & 38.7 & 258.2 & 100.4 & 38.9 \\
5 & 246.1 & 78.9 & 32.5 & 353.3 & 92.8 & 26.3 \\
6 & 188.4 & 65.8 & 34.9 & 373.2 & 104.1 & 27.9 \\
7 & 131.9 & 55.8 & 42.3 & 257.9 & 110.7 & 42.9 \\
8 & 164.3 & 102.3 & 62.2 & 342.1 & 196.7 & 57.5 \\
9 & 198.8 & 84.9 & 42.7 & 375.4 & 111.8 & 29.8 \\
10 & 232.5 & 113.5 & 48.8 & 355.7 & 186.4 & 52.4 \\
11 & 163.7 & 160.2 & 97.9 & 212.1 & 236.6 & 111.5 \\
Mean \pm SD & $189.3 \pm 35.1$ & $90.6 \pm 29.7$ & $48.9 \pm 18.1$ & $330.6 \pm 83.4$ & $136.2 \pm 48.7$ & $44.6 \pm 24.7$ \\
\hline
\end{tabular}

Superscript/subscript characters: $u$ : upper lobe, $l$ : lower lobe, $i$ : inflated state, and $d$ : deflated state. $r$ is the ratio of volume change: $V^{d} / V^{i}$
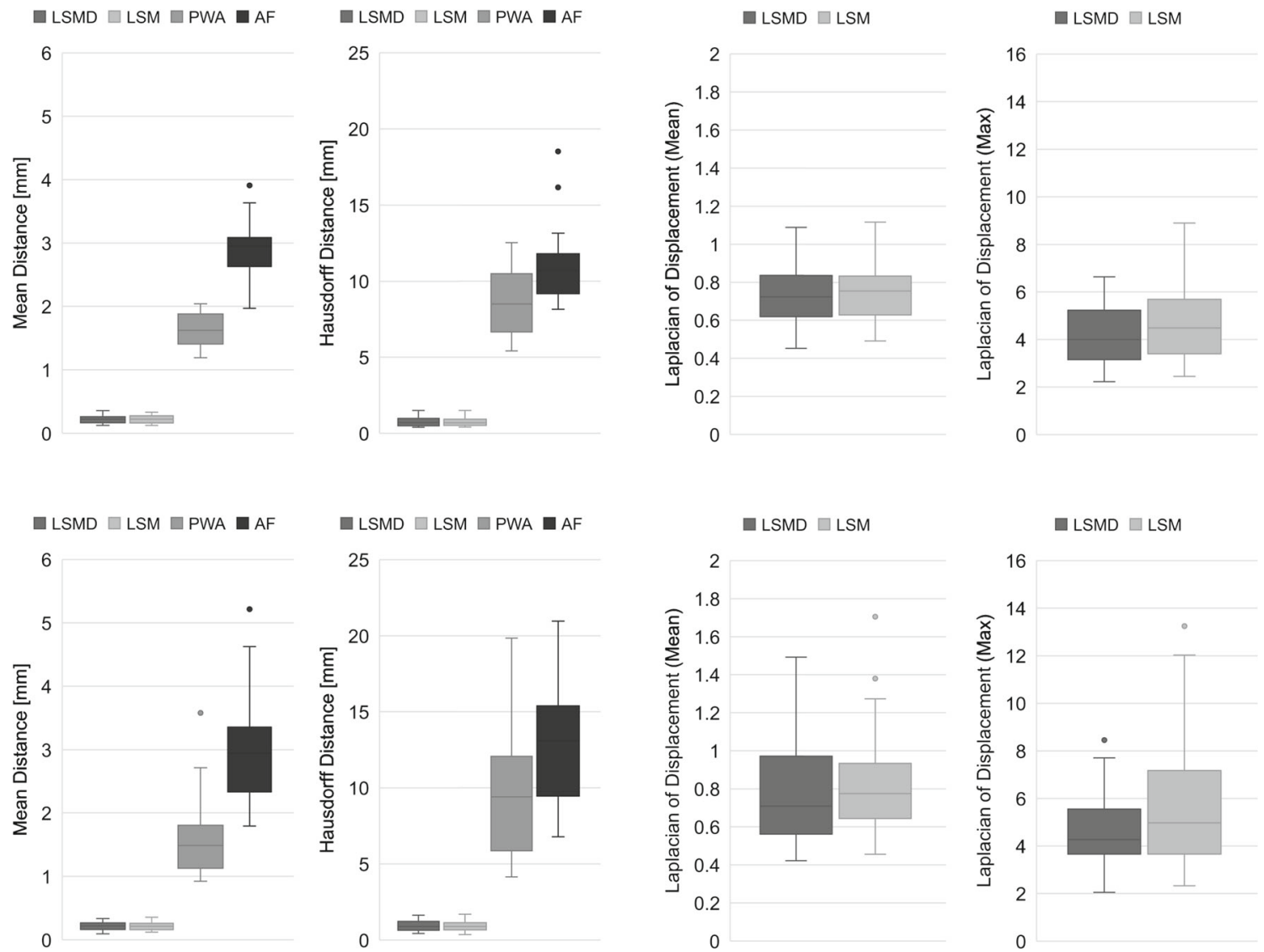

Fig. 5 Quantitative comparison results: mean distance, Hausdorff distance, and Laplacian of the displacement (mean and maximum) of shape matching algorithms for inflated (upper row) and deflated (lower row) lungs

evaluated by the target displacement error (TDE) defined in Eq. (7)

$T D E=\left|\hat{\boldsymbol{u}}_{e}-\boldsymbol{u}_{e}\right|$

where $\boldsymbol{u}_{e}$ is the displacement vector computed from the pair of the corresponding vertex of the registered inflated lung surface and deflated surface, and $\hat{\boldsymbol{u}}_{e}$ is the ground truth displacement obtained from corresponding evaluation points. In this study, the evaluation points were manually placed at the three tip regions and the three midpoints on ridges in the upper/lower lobes, as shown in Fig. 6a, where relatively large deformation and incorrect matching can be assumed. 
Table 2 The average (minimum and maximum) values of all shape matching results for mean distance (MD), Hausdorff distance (HD), Laplacian of displacements (LD), and target displacement error (TDE)

\begin{tabular}{|c|c|c|c|c|}
\hline \multirow[t]{2}{*}{ Metic } & \multicolumn{4}{|l|}{ Methods } \\
\hline & LSMD & LSM & PWA & $\mathrm{AF}$ \\
\hline MD & $0.22(0.10-0.36)$ & $0.22(0.12-0.36)$ & $1.6(0.92-3.58)$ & $3.0(1.79-5.21)$ \\
\hline $\mathrm{HD}[\mathrm{mm}]$ & $0.85(0.39-1.63)$ & $0.82(0.36-1.70)$ & $9.16(4.14-19.8)$ & $12.0(6.78-21.0)$ \\
\hline LD (mean) [mm] & $0.76(0.42-1.49)$ & $0.79(0.45-1.70)$ & & \\
\hline LD (max) [mm] & $4.45(2.05-8.45)$ & $5.19(2.33-13.2)$ & & \\
\hline TDE [mm] & $4.34(0.54-14.0)$ & $5.56(0.5-19.5)$ & $7.0(0.44-25.8)$ & $9.70(1.5-27.9)$ \\
\hline
\end{tabular}

The TDE for LSMD has significantly lower values than the other three methods ( $p$ value $<0.05$ for ANOVA comparing LSMD with LSM, PWA, and AF)

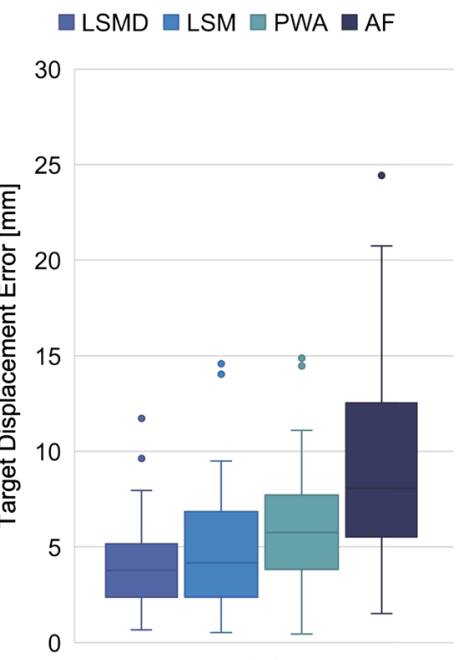

(b)

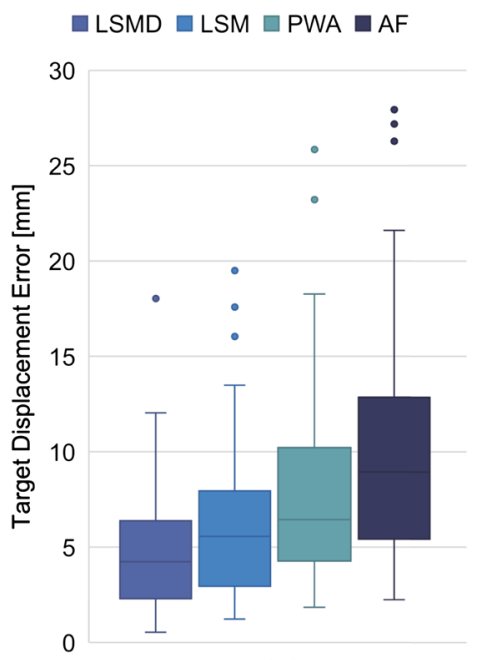

(c)

Fig.6 Quantitative comparison results of target displacement errors (TDE). a The 12 evaluation points manually indicated at the tip or ridge region in upper/lower lobes, $\mathbf{b}, \mathbf{c}$ box plots of TDEs on upper and lower lobes respectively

Figure $6 \mathrm{~b}, \mathrm{c}$ shows the results of the quantitative performance analysis of the registration accuracy on the datasets of the 11 subjects. The error in the lower lobes was larger than that in the upper lobes, which is consistent with the anatomical characteristics, with the volume change and deformation of the lower lobes being generally large. The average, minimum, and maximum of the TDEs of all datasets for LSMD, LSM, PWA, and AF are summarized in Table 2. The TDE of LSMD was significantly lower than that of the other three methods ( $p$ value $<0.05$ for ANOVA test comparing LSMD with LSM, PWA, and AF). The results show that the proposed method can provide localized displacement with an error within $5 \mathrm{~mm}$ that it can overcome the instability problem inherent in Laplacian-based shape matching of distant structures and that it performs well for large-scale deformations. We note that TDE strictly evaluates the point-to-point correspondence and generally shows larger values than Hausdorff distance. Specifically, as in this study, the evaluation points were located at the tips or ridges of the lobes, and the registration error in the other areas is expected to be smaller than that defined by these TDEs.

\section{Pneumothorax deformation analysis}

\section{Mean and variation of deformation}

No study has investigated the impact of inter-subject variation on pneumothorax deformation. Our shape matching framework can directly provide a statistical representation of the registered lung models $\left(M_{I}^{(k)}, M_{D}^{(k)}\right)$, which represents the mean and variation of the pneumothorax deformation between subjects.

Figure 7 shows the deformation modes that correspond to the first two eigenvalues of the obtained pneumothorax deformation models $D^{(k)}$. The eigenvalues and eigenvectors were computed from the set of displacement vectors of all vertices based on singular value decomposition. Five images were generated by changing the weights to plus/minus two times the squared root of the eigenvalues. Please see the supplemental movies that visualize the sequential motion expressed by interpolation of the inflated and deflated states. The color map shows the spatially distributed magnitude of the displacements $\boldsymbol{u}_{i}$ between two surfaces. For example, the surfaces in the mean deformation model deform from 0-32 $\mathrm{mm}$. The 


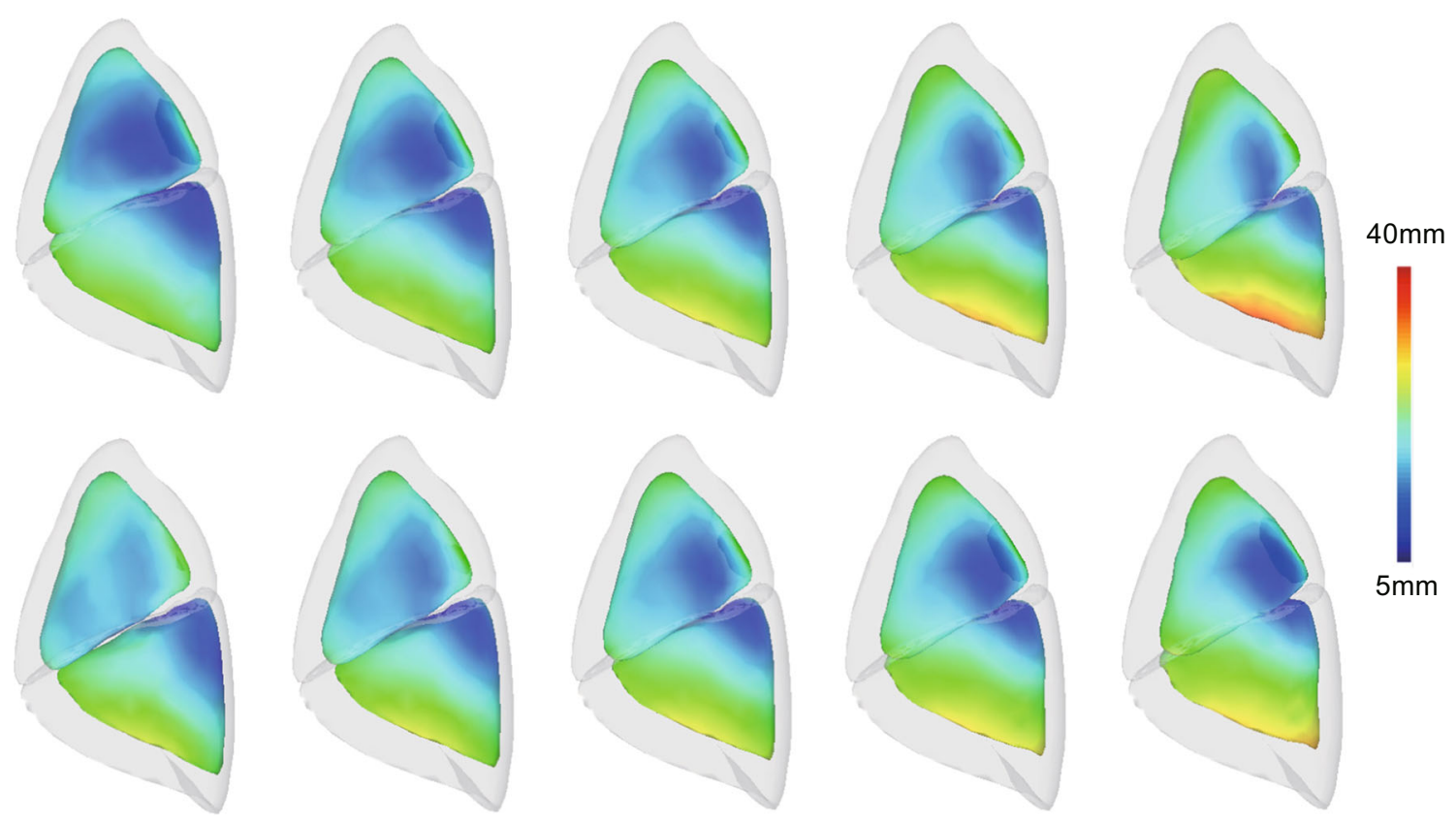

Fig. 7 Visualizations of deformation variations corresponding to the first two eigenvalues of the obtained pneumothorax deformation models. The color map shows the magnitude of the displacements between inflated and deflated lungs. Mean deformation is located in the middle

types of variation in the pneumothorax deformation can be confirmed according to their morphological properties as follows:

- The first eigenvector mainly encompasses variation in the scale of the deformation.

- The second eigenvector is associated with the directions and rotations of the deformation.

We also confirmed that the subspace representation using two eigenvectors explains $96.5 \%$ of the total deformation variation.

\section{Linearity of deformation}

Figure 8 shows the shape matching results and the relationship between the Euclidean distance from the pulmonary hilum of the upper/lower lobes and the magnitude of the displacement at all vertices composing the ten lung models (Cases 1-10). Case 11 was removed from this analysis because of incorrect pressure control during CT imaging. The number of the plots in the graph equals the number of vertices in the registered template model. The graphs show that the relationship between the distance and displacement is mostly linear; however, the displacement in the region distant from the hilum tends to be large and nonlinear. Several cases (e.g., Cases 3, 5, 8, and 9) present more complex patterns with the plots being split into two branches; this means that the deformation contains rotational components around the pulmonary hilum (as a rotation center) or bending, as shown in the right image of Fig. 1. These findings suggest that the pneumothorax deformation modeling requires both global shape changes with the assumption of linearity, and subject-specific physical interactions or boundary conditions between the lobes and the thoracic cavity.

\section{Discussion}

To our knowledge, this study is the first to show the impact of spatial displacement on pneumothorax deformation of in vivo whole lungs within a 5-mm registration error. Past analyses have mainly focused on the internal structures of lungs measured using CBCT, and existing registration methods tended to result in large registration errors, specifically around the tips of the lobes. By integrating optimization of a differential displacement field into Laplacian-based shape matching, the proposed framework addresses problems with matching surfaces that have large curvatures and deformations and thereby achieved smaller registration errors.

To clarify the focus of this research, the displacement of the internal structures was considered to be outside the scope of this paper. Our experiments concentrated on calculation and analysis of the spatial displacements of lung surfaces, including the tips of the upper/lower lobes with large curvatures. In vivo data analysis of the internal structures of inflated/deflated lungs was reported in [26]. Non-rigid registration of lungs deformed by the patients' postures was also investigated in [25]. For clinical applications, intraoperative iatrogenic manipulation, which can result in considerable 


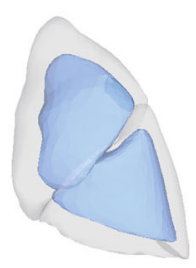

Case 1
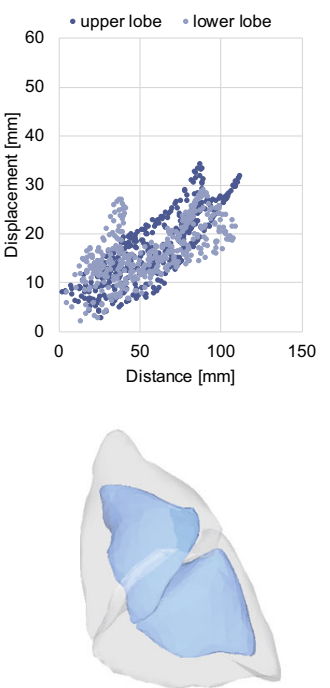

Case 6

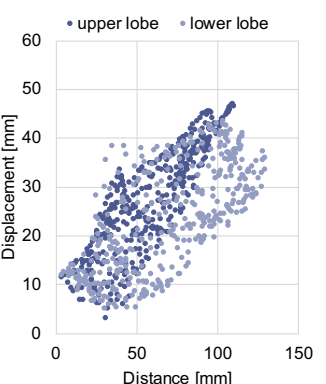

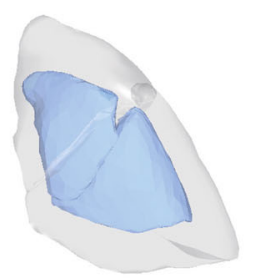

Case 2
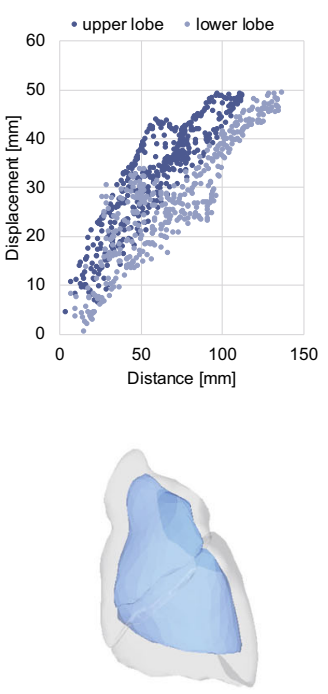

Case 7

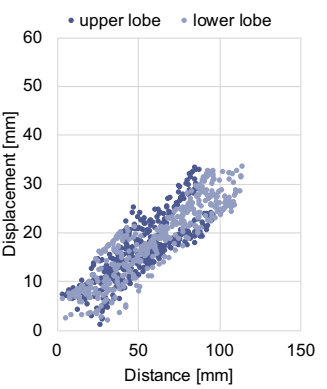

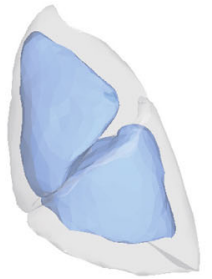

Case 3
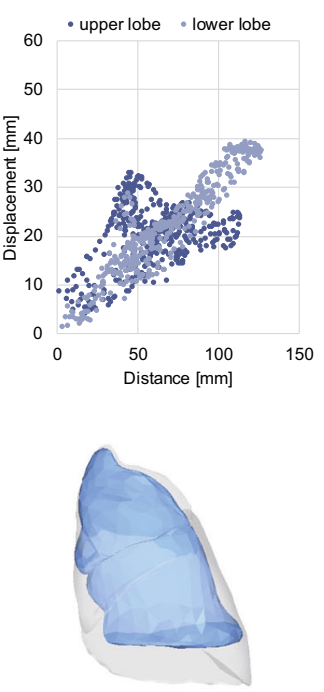

Case 8

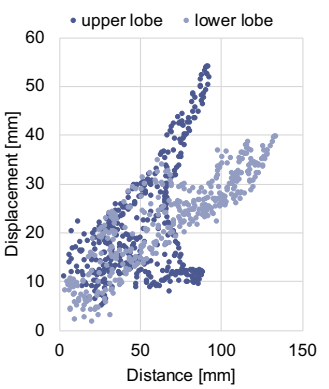

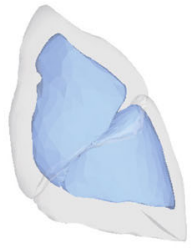

Case 4
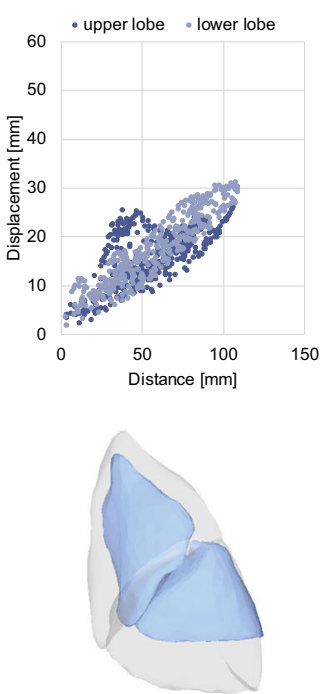

Case 9

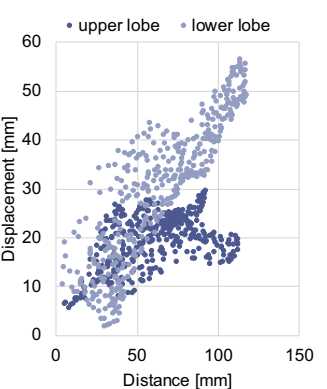

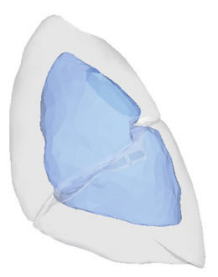

Case 5
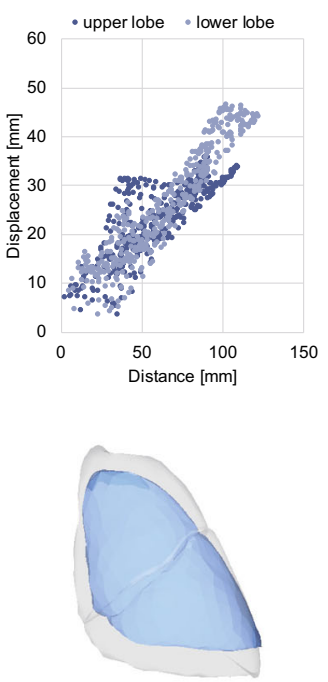

Case 10

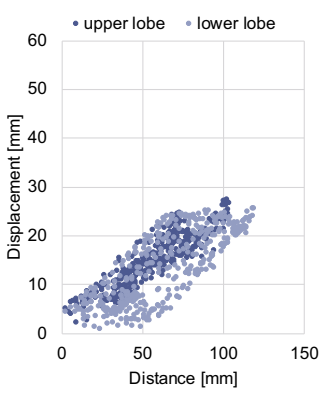

Fig. 8 Shape matching results and the relationship between surface displacement and Euclidean distance from the hilum. The translucent image shows the inflated state and the opaque image the deflated state. In the scatter plot, the light blue and dark blue plots are the displacements of the upper lobe and lower lobe, respectively

evaluate the effectiveness of dense pixel information. As mentioned in "Introduction" section, CT intensity and image contrast changes between the inflated and deflated states are caused by differences in the air content of the lung parenchyma, which is a drawback of image-based registration. However, recent studies have reported that regularized keypoint matching improves deformable registration in lung CT and shows good scores for COPD registration [13]. Learning-based methods using convolutional networks [40] might be useful for registering subject-specific large deformations. We note that the computation time for registering each lobe was $67.6 \pm 16.5 \mathrm{~s}$. The calculation cost of searching for the positional constraints is high, which restricts the number of vertices in the template. It would be interesting to integrate newer image-based registration concepts with 
shape matching and to evaluate their performance with the measured pneumothorax data, with the goals of facilitating precise statistical modeling and improving computation time.

Regarding the limitations of the validation protocol, surface-to-surface measures such as the Hausdorff and mean distances may be insufficient to evaluate local correspondence, especially in cases where the posture of the lobes varies greatly in the temple, or in low-curvature areas where the vertices are sparsely placed. In addition, target displacement error was evaluated on manually selected points in the high curvature areas. We consider that a further exploration of better validation protocols, such as a phantom study, is needed for more reliable evaluation.

In our experiments, the imaging data were only collected from 11 subjects, and further acquisitions were difficult because of renovations to our animal experiment facilities. Because intraoperative CT imaging is not a standard clinical protocol in thoracoscopic surgery, it is not easy to construct a patient-specific image database of collapsed lungs. However, despite this study's limited data size, the results show that variations in pneumothorax deformation are not large. This suggests that even if there are variations in the shapes and volumes of individual lungs in the inflated state, subject-specific deformation can be formulated with relatively customized nonlinear models. This paper targeted the left lung, to measure stable pneumothorax deformation from the limited number of live dogs available. In the right lung, the physical interaction between the three (upper, middle, and lower) lobes and the ventilator may be more complex than that in the left lung. As the same measurement protocol and registration algorithms can be applied to right lungs, further research to develop statistical models of right lungs is desirable.

\section{Conclusion}

This study aimed to analyze three-dimensional surface displacement in pneumothorax deformation using model-based shape matching techniques. To perform shape matching for substantially deformed lung images, a complete Laplacianbased shape matching solution that optimizes the differential displacement field was introduced. Our experiments showed that the proposed concept addresses problems with matching surfaces that have large curvatures and deformations, and that it achieved smaller registration errors than other techniques, even at the tip region, with spatial displacement of the lung's surface being visualized within a 5-mm error. The findings and inter-subject statistical representations obtained in this study are directly available for further research on pneumothorax deformation modeling. In future work, we will explore deformation estimation methods and develop an intraoperative guidance system for VATS.
Acknowledgements This research was supported by the Japan Agency for Medical Research (AMED) and the Acceleration Transformative Research for Medical Innovation (ACT-M) Program. A part of this study was also supported by a JSPS Grant-in-Aid for challenging Exploratory Research (grant number 18K19918). We thank Kotaro Kobayashi for preprocessing image data and thank Richard Lipkin, $\mathrm{PhD}$, from Edanz Group (www.edanzediting.com/ac) for editing a draft of this manuscript.

\section{Compliance with ethical standards}

Conflict of interest The authors declare that they have no conflict of interest.

Ethical approval All applicable international, national, and/or institutional guidelines for the care and use of animals were followed. All procedures performed in studies involving animals were in accordance with the ethical standards of the institution or practice at which the studies were conducted. This article does not contain any studies with human participants performed by any of the authors.

Open Access This article is distributed under the terms of the Creative Commons Attribution 4.0 International License (http://creativecomm ons.org/licenses/by/4.0/), which permits unrestricted use, distribution, and reproduction in any medium, provided you give appropriate credit to the original author(s) and the source, provide a link to the Creative Commons license, and indicate if changes were made.

\section{References}

1. Shaw JP, Dembitzer FR, Wisnivesky JP, Litle VR, Weiser TS, Yun J, Chin C, Swanson SJ (2008) Video-assisted thoracoscopic lobectomy: state of the art and future directions. Ann Thorac Surg 85(2):S705-S709

2. Flores RM, Alam N (2008) Video-assisted thoracic surgery lobectomy (VATS), open thoracotomy, and the robot for lung cancer. Ann Thorac Surg 85(2):S710-S715

3. Sato M, Omasa N, Chen F, Sato T, Sonobe M, Bando T, Date H (2014) Use of virtual assisted lung mapping (VAL-MAP), a bronchoscopic multispot dye-marking technique using virtual images, for precise navigation of thoracoscopic sublobar lung resection. J Thorac Cardiovasc Surg 147(6):1813-1819

4. Lin MW, Chen JS (2016) Image-guided techniques for localizing pulmonary nodules in thoracoscopic surgery. $\mathrm{J}$ Thorac Dis 8(9):S749-S755

5. Sotiras A, Davatzikos C, Paragios N (2013) Deformable medical image registration: a survey. IEEE Trans Med Imaging 32(7):11531190

6. Oh S, Kim S (2017) Deformable image registration in radiation therapy. Radiat Oncol J 35(2):101-111

7. Beg MF, Miller MI, Trouve A, Younes L (2005) Computing large deformation metric mappings via geodesic flows of diffeomorphisms. Int J Comput Vision 61(2):139-157

8. Kenngott HG, Wagner M, Gondan M, Nickel F, Nolden M, Fetzer A, Weitz J, Fischer L, Speidel S, Meinzer HP, Bockler D, Buchler MW, Müller-Stich BP (2014) Real-time image guidance in laparoscopic liver surgery: first clinical experience with a guidance system based on intraoperative CT imaging. Surg Endosc 28(3):933-940

9. Nickel F, Kenngott HG, Neuhaus J, Sommer CM, Gehrig T, Kolb A, Gondan M, Radeleff BA, Schaible A, Meinzer HP, Gutt CN, Müller-Stich BP (2013) Navigation system for minimally inva- 
sive esophagectomy: experimental study in a porcine model. Surg Endosc 27(10):3663-3670

10. Wagner M, Gondan M, Zollner C, Wunscher JJ, Nickel F, Albala L, Groch A, Suwelack S, Speidel S, Maier-Hein L, Müller-Stich BP, Kenngott HG (2016) Electromagnetic organ tracking allows for real-time compensation of tissue shift in image-guided laparoscopic rectal surgery: results of a phantom study. Surg Endosc 30(2):495-503

11. Nickel F, Kenngott HG, Neuhaus J, Andrews N, Garrow C, Kast J, Sommer CM, Gehrig T, Gutt CN, Meinzer HP, Müller-Stich BP (2018) Computer tomographic analysis of organ motion caused by respiration and intraoperative pneumoperitoneum in a porcine model for navigated minimally invasive esophagectomy. Surg Endosc 32(10):4216-4227

12. Koo B, Özgür E, Le Roy B, Buc E, Bartoli A (2017) Deformable registration of a preoperative $3 \mathrm{D}$ liver volume to a laparoscopy image using contour and shading cues. In: Medical image computing and computer assisted intervention (MICCAI), 326-334

13. Rühaak J, Polzin T, Heldmann S, Simpson IJA, Handels H, Modersitzki J, Heinrich MP (2017) Estimation of large motion in lung CT by integrating regularized keypoint correspondences into dense deformable registration. IEEE Trans Med Imaging 36(8):17461757

14. Nakao M, Minato K (2010) Physics-based interactive volume manipulation for sharing surgical process. IEEE Trans Inf Technol Biomed 14(3):809-816

15. Nakao M, Oda Y, Taura K, Minato K (2014) Direct volume manipulation for visualizing intraoperative liver resection process. Comput Methods Progr Biomed 113(3):725-735

16. Gunay G, Ha LM, Walsum TV, Klein S (2016) Semi-automated registration of pre- and intra-operative liver CT for image-guided interventions. In: SPIE medical imaging: $97841 \mathrm{~N}$

17. Sadeghi-Naini A, Shirzadi Z, Samani A (2012) Towards modeling tumor motion in the deflated lung for minimally invasive ablative procedures. Comput Aided Surg 17(5):211-220

18. Ilegbusi OJ, Li Z, Seyfi B, Min Y, Meeks S, Kupelian P, Santhanam AP (2012) Modeling airflow using subject-specific 4DCTbased deformable volumetric lung models. Int J Biomed Imaging 2012:350853

19. Fuerst B, Mansi T, Carnis F, Salzle M, Zhang J, Declerck J, Boettger T, Bayouth J, Navab N, Kamen A (2015) Patient-specific biomechanical model for the prediction of lung motion from 4-D CT images. IEEE Trans Med Imaging 34(2):599-607

20. Wilms M, Ha IY, Handels H, Heinrich MP (2016) Model-based regularisation for respiratory motion estimation with sparse features in image-guided interventions. In: Medical image computing and computer-assisted intervention (MICCAI), 89-97

21. Nakao M, Kawashima A, Minato K, Kokubo M, (2007) Simulating lung tumor motion for dynamic tumor-tracking irradiation. In: IEEE nuclear science symposium and medical imaging conference, 4549-4551

22. Naini AS, Pierce G, Lee TY, Patel RV, Samani A (2011) CT image construction of a totally deflated lung using deformable model extrapolation. Med Phys 38(2):872-883

23. Nakamoto M, Aburaya N, Sato Y, Konishi K, Yoshino I, Hashizume M, Tamura S (2007) Thoracoscopic surgical navigation system for cancer localization in collapsed lung based on estimation of lung deformation. In: Medical image computing and computer-assisted intervention (MICCAI), 68-76

24. Wu S, Nakao M, Matsuda T (2017) Continuous lung region segmentation from endoscopic images for intra-operative navigation. Comput Biol Med 87(1):200-210

25. Alvarez P, Chabanas M, Rouze S, Castro M, Payan Y, Dillenseger JL (2018) Lung deformation between preoperative CT and intraoperative CBCT for thoracoscopic surgery: a case study. In: SPIE medical imaging, 105761D
26. Uneri A, Nithiananthan S, Schafer S, Otake Y, Stayman JW, Kleinszig G, Sussman MS, Prince JL, Siewerdsen JH (2013) Deformable registration of the inflated and deflated lung in cone-beam CTguided thoracic surgery: initial investigation of a combined modeland image-driven approach. Med Phys 40(1):017501

27. Heiselman JS, Collins JA, Clements LW, Weis JA, Simpson AL, Geevarghese SK, Kingham TP, Jarnagin WR, Miga MI (2018) Technical note: nonrigid registration for laparoscopic liver surgery using sparse intraoperative data. In: Proceedings SPIE Medical Imaging, 105760D

28. Saito A, Nakao M, Uranishi Y, Matsuda T (2015) Deformation estimation of elastic bodies using multiple silhouette images for endoscopic image augmentation. In: IEEE international symposium on mixed and augmented reality, 170-171

29. Nakao M, Saito A, Matsuda T (2017) A simulation study on deformation estimation of elastic materials using monocular images. Int J Comput Assist Radiol Surg 12(1):S257-258

30. Klein S, Staring M, Murphy K, Viergever MA, Pluim JPW (2010) Elastix: a toolbox for intensity based medical image registration. IEEE Trans Med Imaging 29(1):196-205

31. Ehrhardt J, Werner R, Schmidt-Richberg A, Handels H (2011) Statistical modeling of $4 \mathrm{D}$ respiratory lung motion using diffeomorphic image registration. IEEE Trans Med Imaging 30(2):251-265

32. Kim J, Valdes-Hernandez Mdel C, Royle NA, Park J (2015) Hippocampal shape modeling based on a progressive template surface deformation and its verification. IEEE Trans Med Imaging 34(6):1242-1261

33. Jud C, Giger A, Sandkhler R, Cattin PC (2017) A localized statistical motion model as a reproducing kernel for non-rigid image registration. In: Medical image computing and computer-assisted intervention (MICCAI), 261-269

34. Kazhdan M, Bolitho M, Hoppe H (2006) Poisson surface reconstruction. In: Symposium on geometry processing, 61-70

35. Sorkine O, Cohen-Or D, Lipman Y, Alexa M, Rössl C, Seidel HP (2004) Laplacian surface editing. In: Proceedings of the 2004 eurographics/ACM SIGGRAPH symposium on geometry processing, $175-184$

36. Nealen A, Igarashi T, Sorkine O, Alexa M (2006) Laplacian mesh optimization. In: Proceedings of the 4th international conference on computer graphics and interactive techniques in Australasia and Southeast Asia, 381-389

37. Huttenlocher D, Klanderman G, Rucklidge W (1993) Comparing images using the Hausdorff distance. IEEE Trans Pattern Anal Mach Intell 15:850-863

38. Kobayashi K, Nakao M, Tokuno J, Chen TF, Matsuda T (2018) Analysis of deaeration deformation in ex vivo animal lung by Laplacian-based surface registration. In: 40th Annual international conference of the IEEE engineering in medicine and biology society $(\mathrm{EMBC})$

39. Zhou J, Kim S, Jabbour S, Goyal S, Haffty B, Chen T, Levinson L, Metaxas D, Yue NJ (2010) A 3D global-to-local deformable mesh model based registration and anatomy-constrained segmentation method for image guided prostate radiotherapy. Med Phys 37:1298-1308

40. Boscaini D, Masci J, Melzi S, Bronstein MM, Castellani U, Vandergheynst P (2015) Learning class-specific descriptors for deformable shapes using localized spectral convolutional networks. Comput Graph Forum 34(5):13-23

Publisher's Note Springer Nature remains neutral with regard to jurisdictional claims in published maps and institutional affiliations. 